\title{
Harnessing Pharmacogenomics to Tackle Resistance to the "Nucleoside Reverse Trancripatse Inhibitor” Backbone of Highly Active Antiretroviral Therapy in Resource Limited Settings
}

\author{
Misaki Wayengera ${ }^{*}, 1,2$, Henry Kajumbula ${ }^{2}$ and Wilson Byarugaba ${ }^{1,3}$ \\ ${ }^{I}$ Division of Human and Molecular Genetics-Dept of Pathology College of Health Sciences, Makerere University P.O. \\ Box 7072, Kampala, Uganda \\ ${ }^{2}$ Division of Molecular Biology Dept of Microbiology College of Health Sciences, Makerere University P.O. Box 7072 \\ Kampala, Uganda \\ ${ }^{3}$ Kampala International University School of Health Sciences, Uganda
}

\begin{abstract}
Background: The sustainable use of HAART within the sub-Saharan and other developing world settings faces the emerging challenge of drug resistance. Nucleoside reverse transcriptase inhibitors (NRTI) form the backbone of HAART and preserving their "antiviral efficacy" is thus critical to sustainable HAART use.

Methods: A systematic review of the "mechanisms of evolution" of resistance to NRTI at the HIV genome level, and the phenotypic manifestations on drug pharmacokinetics was done.

Conclusion: This paper provides an evidence based account of how the knowledge of pharmacogenomics may be exploited to tackle NRTI resistance within limited resource.
\end{abstract}

Keywords: Acquired immunodeficiency syndrome(AIDS), highly active antiretroviral therapy (HAART), human immunodeficiency virus (HIV), drug resistance.

\section{INTRODUCTION}

The highest burden of the human immunodeficiency virus (HIV) epidemic is concentrated in the sub-Saharan region. Over $70 \%$ of all global HIV infections have been found to occur here [1]. Despite the earlier policy and patent controversies surrounding the use of highly active antiretroviral therapy (HAART) within this setting, HAART has widely gained application here [2]. This access to HAART can be mainly attributed to several advocacy and funding avenues [2-4]. Specifically, the World Bank and its global partners, in particular, with commitment by the G8, have ensured that several countries within this setting can meet the WHO 3'by 5 " target of treating 3 million by $2005[3,4]$. Through Initiative such as the global Fund to Fight AIDS, TB and MalariaGFATM, the President's Emergency Plan for AIDS Relief (PEPFAR) and several others, about 2 million persons living with HIV/AIDS are accessing HAART today [2, 3]. The evolution of resistance however poses one of the biggest challenges to HAART use here. Developing mechanisms to curtail its growing prevalence amidst the existent limited resources may be more cost effective and realistic than waiting to deal with an outright "outbreak" of resistant virus. One way to do so is by ensuring that all persons living with HIV or the Acquired Immunodeficiency Syndrome (AIDS):

*Address correspondence to this author at the Division of Human and Molecular Genetics-Dept of Pathology College of Health Sciences, Makerere University P.O. Box 7072, Kampala, Uganda; Tel: +256782450610;

E-mail:wmisaki@yahoo.com
PLWHA on HAART take their medications in the right dosages and at the right time. This is because non compliance is one of the major factors that influence the evolution of resistance [5]. The second option would be to develop the necessary capacity for monitoring drug sensitivity and profiling resistance. The latter option has however remained widely unavailable at several of the HIV/AIDS treatment and care centers- mainly due to the high costs that are involved in phenotyping and genotyping HIV resistance to HAART [2]. To ensure the success of the HAART programs in this setting however, the capacity to deal with resistance to HAART is needed as an integral part of the HIV treatment and care systems here, emphasizing why global partners in the fight against the HIV epidemic should consider a focused look at the subject. The third option that is widely explored elsewhere but is still limited in the developing world is the use of "salvage regimens" [6].

Amidst the above available options, until such a time when capacity for resistance profiling and access to equally diverse options for "salvage therapy" become readily available in this setting, it is the responsibility of both the PLWHA and the respective primary care physicians to work together so as to preserve current first line HAART options from resistance. Several compliance studies have shown that sub-Saharan PLWHA are doing their part relatively well in terms of compliance to HAART [5].

Physicians, through knowledge of HAART pharmacogenomics, specifically, the effects of individual resistance phenotypes on overall HAART pharmacodynamics (PD) and 
pharmacokinetics (PK), may equally play a significant part in setting a barrier to the evolution of resistance to first line HAART options. By so doing, they can as well devise virologically effective options for "salvage therapy" from the limited options of HAART available. Today, according to the International AIDS Society, the ideal combination of HAART should comprise a backbone of two NRTIs plus either a Protease Inhibitor or a Non-NRTI (NNRTI). NRTIs thus form a significant component of HAART whose function should be jealously "guarded" to ensure sustainable use of any selected HAART regimen or combination. This paper aims to examine how "evolution of resistant phenotypes and genotypes to NRTI (and NNRTI) influences the general PD and PK of HAART". From that examination, we hope to provide an evidence based account of how knowledge of this "pharmacogenomics" may be applied in resource limited setting to prevent or delay the evolution of resistance to HAART while maintaining virologic suppression among those with identified resistance.

\section{GENERAL CONCEPT OF HOW RESISTANCE EMERGES IN HAART AND ITS IMPLICATION ON FIGHTING RESISTANCE TO HAART}

\section{The Evolution of Resistance to HAART: A Genomic Perspective}

Drug resistance to HAART in general results from mutations in the viral genome [6]. Specifically, those mutations associated to HAART resistance are localized on those genes that encode the molecular targets of antiretroviral drugs. Hence resistance to NRTI/NNRTI, Protease inhibitors (PIs) and fusion inhibitors can be localized on reverse transcriptase, protease, and glycoprotein 41 respectively. Well as these mutations have been noted to occur spontaneously in the HIV genome, and resistant phenotypes exist even prior to the use of HAART (implying that the use of HAART in itself does not select for resistant virus)[6], it has equally been observed that the propagation and maintenance of these resistant or mutant viruses is largely favored by sub-optimal plasma inhibitory concentrations (PIC) of the HAART component to which resistance exists. The latter serves to kill off the non resistant viruses (which are still susceptible to the combination of HAART in use) and leaves the mutant to shrive. In general, for any organism or pathogen- the mutation rate is a dependant on three factors: (a) Presence of an outside selective pressure-which for microbes like HIV is often a drug that is present at lower than minimal inhibitory or lethal dosages to the microbe (b) frequency of replication or duplication of the microbe and (c) efficacy of the proof reading mechanisms of the process of DNA or RNA synthesis during replication. Because of the rapid replication rate of HIV (where the virus produces $10^{10}$ billion copies every 24 hours), and the error prone reading mechanism of the RNA polymerase relative to its DNA counterpart (DNA polymerase), HIV is inherently highly susceptible to variations in the genotypes; meaning that even the slightest pressure from a misuse of drug combinations is likely to result into the evolution of resistance due to an" inherent narrow viral resistance barrier" [6, 7].

\section{Phenotypic Manifestation of Resistant Genotypes and the Mechanisms of Resistance}

Most NRTI are analogues of deoxynucleoside triphosphates (dNTs) that are integrated into the DNA (or RNA) chain in the same way that normal dNTs are during the processes of DNA or RNA synthesis. However, because of alterations in the configuration of the NRTIs, NRTIs can not be processed further by the DNA or RNA synthesizing enzymes_ (DNA polymerase and RNA polymerase respectively). This results into premature termination of the nucleotide chain synthesis, thus respective cell proliferation [6]. NRTIs specifically target function of viral reverse transcriptase enzyme. Two Mechanism of resistance against reverse transcriptase inhibitors are known. These are:

1. Discrimination: This occurs when the reverse transcriptase (RT) enzyme develops the ability to evade binding of the NRTI or NNRTI but retains its ability to bind the natural deoxynucleoside triphosphates substrates. Point mutations such as K65R and M184V are associated with this phenotype [6].

2. Pyrophosphoroylation: This involves an increased phosphorolytic removal of the chain terminating NRTI from the 3'end of the primer after it has been integrated into viral DNA strand. This mechanism, that is also called "primer unblocking" or "excision" is mainly associated with thymidine analogue mutations (TAMs) selected for by Stavudine and $\mathrm{Zi}-$ dovudine (M41L, T215Y/F and K218Q/E) [6,7].

Although different drugs select for different mutations associated with different resistance profiles or mechanisms, what is of clinical significance in dealing with resistant virus is how the evolution of resistant phenotypes may be avoided, or dealt with.

To do so, we find that one needs to appreciate the pharmacogenomics of HAART resistance, particularly (1) what mutations (genotypes) are associated with misuse of particular NRTIs (or their combinations) and (2) the possible beneficial effects of those resultant phenotypes on the PK and PD of other HAART components.

\section{PHENOTYPIC MANIFESTATIONS OF RESISTANT GENOTYPES ASSOCIATED WITH SPECIFIC NRTI (OR COMBINATION OF NRTIS) OF RELEVANCE TO DEALING WITH RESISTANCE IN RESOURCE LIMITED SETTINGS}

Three main "combinations of two "NRTIs" have gained wide usage globally as backbones in HAART; and are equally used within the sub-Saharan setting. These are Tenofovir/Emtricitabine (Truvuda), Zidovudine/lamivudine (combivir) and Abacavir/lamivudine (Kivexa)[2]. Most other combinations used as backbones involve an interchange of the individual drugs in these three. When virologic failure is observed in patients using any of these, it may be due to resistance to a single component of HAART or all. To note is that: some drug combinations are associated with "much narrower resistance barrier than others. In addition, while the occurrence of a "single mutation" may suffice to confer resistance to some drugs that comprise HAART regimens such 
as is the case with M184V/L74V for lamivudine (3TC) and Emtricitabine (FTC); other drugs such as Zidovudine (AZT) and Stavudine (d4T) have a broader barrier to resistance and an accumulation of "resistance mutations" must occur for phenotypic resistance to manifest [6]. In any patient on a specified combination of HAART, various populations of mutant viruses with differing susceptibility to individual drug components of the HAART regimens co-exist. Some of these mutant genotypes such as several Thymidine Analogue Mutations (TAMs) may confer resistances to all members of the group as related above, while some resistant genotypes are only associated with resistance to a single member of the group such as the signature mutations of Tenofovir(K65R); with limitations the two mutations M184V and L74V that are often associated with sub-optimal use of and lamivudine and Abacavir [8-10]. Specifically, while the insertions in codon 69 and 70(T69) has been found to be associated with a high resistance to ZDV, d4T, ddI, ABC and TDF [11-13], the mutation in the conserved domain of RT-Q151M; whose occurrence has been associated with two drug therapy_ didanosine with either Zidovudine or Stavudine( which is a rare scenario in modern HAART combining 3 drugs) confers resistance to all NRTIs [13]. Some mutations associated with resistance to certain drugs such as the K65R and M184V mutations for Tenofovir and Lamivudine/Abacavir respectively have been found to confer an increased susceptibility to other NRTIs like Zidovudine [11,12]. In other words, while predominance of some mutants associated with resistance to a given single member of the NRTI-backbone confers resistance to all members of the group, some mutants are associated with phenotypic manifestations that confer an increased susceptibility to another NRTI. The significance of these points above when dealing with clinical resistance to HAART in a resource limited setting is discussed below.

\section{EXPLOITING NRTI RESISTANCE PHARMACOGE- NOMICS TO PREVENT THE EVOLUTION OR DEAL WITH EXISTENT NRTI RESISTANCE IN A RE- SOURCE POOR SETTINGS}

Marcelin AG et al. [6] and others have provided a critical review of the phenotypic manifestations of NRTI resistance genotypes [9-18], the fitness between mutant and wild type viruses [6], and inherent "resistance barriers" for individual NRTIs [9-18]. Specifically, the review by Marcelin AG et al. [6] reveals a number insight into the beneficial "pharmacokinetics" of NRTI resistance which may be applied to prolong HAART sustainability in resource limited settings by improving the general outcome of NRTIs. These benefits are and can be accrued as detailed in the three scenarios here.

First, using high resistance barrier first line combinations (while avoiding those with low resistance barriers); it is possible to delay the emergence of resistant mutants to an HAART combination. This serves the purpose of preserving future options. For instance, lets consider the 3TC/FTC plus thymidine analogues scenario. It has been noted that the systematic use of 3TC/FTC plus thymidine analogues have the advantage of low resistance mutations and associated virologic failure, and its use may serve to preserve future options. In so doing, there is however need to avoid prolonged viral replication by using either a NNRTI or Protease inhibitor (PI) in addition [2,9]. Another scenario is that of $\mathrm{ABC} / 3 \mathrm{TC}$ plus NRTI with NNRTI or PI. This combination of NRTIs similarly serves the purpose of preserving future options as the evolution of the M184V resistance mutation to Abacavir(ABC) serves to increase susceptibility to NRTIs(specifically Zidovudine) while L74V mutation commonly associated with lamivudine misuse only affects didanosine(ddI) among the NRTIs. The lowest mutation rates have been observed when this backbone combination of $\mathrm{ABC} / 3 \mathrm{TC}$ is used in combination with PIs [14]. Lastly, avoiding didanosine with other thymidine analogues is critical, since when thymidine analogues such as Zidovudine (ZDV) and Stavudine (d4T) are used in combination with didanosine, a high rate of thymidine analogue mutations (TAMs) is seen with failure, many of which confer cross resistance to other NRTIs. When used in isolation, didanosine selects for L74V mutation and more rarely the K65R $[6,15-17]$. While the presence of L74V only affects ddI, when associated with other mutations such as TAMS, L74V confers resistance to TDF and $\mathrm{ABC}$ [6].

Second, by exploring interactive co-potentiation that some mutants associated with resistance to a particular drug confer to in situ bioavailability and viral susceptibility to another, it is possible to enhance viral suppression. One such example is use of TDF with NRTIs. When Tenofovir (TDF) is used in a patient on NRTIs in whom prior TAMs have evolved, the TDF signature mutation K65R emerges [10-13]. However, K65R has the antagonistic effect of reducing the excision process induced by TAMs (thus associated resistance to NRTIs). In addition, biochemical studies have shown a similar antagonism between K65R and L74V that is correlated with a poor ability of the mutant viruses with the double RT resistance mutations "K65R+L74V" to use natural nucleosides relative to wild types [11-13]. As another example, in using 3TC/FTC with Zidovudine, it has been shown that changes in the reverse transcriptase enzyme associate with the resistant mutation to Lamivudine increases the fidelity and diminished processivity of the viral reverse transcriptase enzyme), an effect that serves to decrease resistant viral fitness [18]. When present with Zidovudine associated mutations, M184V however has been found to equally serve to partially restore Zidovudine (ZDV) efficacy and reduce the emergence of TAMs $[18,19]$. This combination of reduced "resistant virus fitness" and increased susceptibility to ZDV restores virologic efficacy of the HAART combination containing 3TC/FTC plus Zidovudine. Lastly, since exposure to sub-optimal plasma inhibitory concentrations of HAART is the main exogenous selective pressure leading to evolution of resistant mutants and wild type virus is relatively more fit than mutant virus in the absence of HAART induced inhibition or suppression, removing "drug" pressure will serve to create an environment in which wild type virus out compete mutant (resistant) virus_ "survival of fittest" [6]. Drug holidays may thus have a role to play in the management of persons living with HIV/AIDS who develop resistance to major NRTI options for HAART combinations within resource limited settings [6]. 


\section{LIMITATIONS OF THESE GUIDELINES: AN UN- CERTAIN FUTURE}

Most of the conclusions made here have been drawn from clinical trial observations [2, 6, 9-18]. With a prolonged use of HAART and evolution of patterns of resistance not currently documented; the standard of care is bound to change. A continued surveillance of the prevailing patterns of resistance mutations, say at a central laboratory, may serve to inform the decision on whether or not these guidelines can still be "generally" applicable.

\section{WAY FORWARD}

Amidst the general lack of resistance phenotyping and genotyping, and limited options for salvage therapy, a minimal but clinically relevant knowledge of the "beneficial interactive phenotypic manifestations of NRTI resistance genotypes, the variation in fitness between mutant and wild type viruses, and inherent "resistance barriers" for individual NRTIs" is critical for primary health care providers using HAART to treat and care for persons living with HIV or AIDS (PLWHA) in resource poor settings to ensure a sustainable use of HAART. A summary of the forwarded options for dealing with NRTI resistance in resource poor settings is shown in Table $\mathbf{1}$.

Table 1.

A. Using high resistance barrier first line combinations (while avoid-
ing those with low resistance) to delay the emergence of resistant
mutants to an HAART combination
1. Using Lamivudine/ Emtricitabine plus a thymidine analogue to pre-
serving future option
2. Using Abacavir/Lamivudine with NRTI plus NNRTI or PI
3. Avoiding Abacavir/Lamivudine (Kivexa) plus Tenofovir
4. Avoiding Zidovudine analogues with didanosine
B. Exploring interactive co-potentiation that some mutants associ-
ated with resistance to a particular drug confer to in situ bioavail-
ability and viral susceptibility to another
1. Tenofovir with NRTIs other than abacavir/lamivudine
2. Lamivudine/Emtricitabine with Zidovudine
C. Drug holidays for selective elimination of resistant virus

\section{CONCLUSION}

Knowledge of the pharmacogenomics of NRTI may be explored to prevent resistance in resource limited settings.

\section{REFERENCES}

[1] UNAIDS. Report on the global HIV-AIDS epidemic: Geneva 2007.

[2] Attaran A. Adherence to HAART: Africans Take Medicines More Faithfully than North Americans PLoS Med 2007; 4(2): e83.

[3] World Health Organization \& Joint United Nations Programmed on HIV/AIDS (UNAIDS). Progress on global access to HIV antiretroviral therapy: a report on "3 by 5" and beyond Geneva, Switzerland: 2006 World Health Organization, Joint United Nations Pro- gramme on HIV/AIDS (UNAIDS):2007 [Internet: Accessed 12 ${ }^{\text {th }}$ Dec 2007]. Available from: http://www.who.int/hiv/fullreport_en_ highres.pdf]

[4] Office of Global AIDS Coordinator. U.S. President's Emergency Plan for AIDS Relief. Making a difference: supporting antiretroviral treatment, Washington, DC: 2006. Office of Global AIDS Coordinator [Internet: Accessed $12^{\text {th }}$ Dec 2007]. Available from: http:/ /www.state.gov/documents/organization/67502.pdf]

[5] Mills EJ, Nachega JB, Buchan I, et al. Adherence to antiretroviral therapy in sub-Saharan Africa and North America: a meta-analysis. JAMA 2006; 296(6): 679-90.

[6] Marcelin AG, Calvez V, Raffi R. A Comprehensive Guide: HIV resistance to reverse transcriptase nucleoside inhibitors, $1^{\text {st }}$ ed, Mediedition: Paris 2007.

[7] Gallant JE, Gerondelis PZ, Wainberg MA, et al. Nucleoside and nucleotide analogue reverse transcriptase inhibitors: a clinical review $\mathrm{f}$ antiretroviral resistance. Antivir Ther 2003; 8: 489-509.

[8] Shirasaka T, Kavlick MF, Ueno T, et al. Emergence of human immunodeficiency virus type 1 variants with resistance to multiple dideoxynucleosides in patients receiving therapy with dideoxynucleosides. Proc Nat Acad Sci USA 1995; 92: 2398-402.

[9] Kuritzkes DR, Bassett RL, Hazelwood JD, et al. Adult ACTG Protocol 306370 Teams Rate of thymidine analogue resistance mutation accumulation with zidovudine- or stavudine-based regimens J Acquir Immune Defic Syndr 2004; 36(1): 600-3.

[10] Wirden M, Marcelin AG, Simon A, et al. Resistance mutations before and after tenofovir regimen failure in HIV-1 infected patients. J Med Virol 2005; 76(3): 297-301.

[11] Parikh UM, Kootnz DL, Chu CK, Scinaz RF, Melllows JW. In vitro activity of structurally diverse nucleoside analogues against human immunodeficiency virus type 1 with K65R mutation in reverse transcriptase. Antimicrob Agents Chemother 2005; 49: 113944.

[12] Deval J, Navarro JM, Selmi B, et al. A loss of viral replicative capacity correlates with altered DNA polymerization kinetics by the human immunodeficiency virus reverse transcriptase bearing the K65R and L74V dideoxynucleosides resistance substitutions. J Biol Chem 2004; 279: 25489- 96.

[13] Henry M, Tourres C, Colson P, Ravaux I, Poizot-Martin I, Tamalet C. Coexistence of the K65R/L74V and/ or K65R/T215Y mutations in the same HIV-1 genome. J Clin Virol 2006; 37: 227-30.

[14] Eron J Jr, Yeni P, Gathe J Jr, et al. KLEAN study team. The KLEAN study of fosamprenavir-ritonavir versus lopinavirritonavir, each in combination with abacavir-lamivudine for initial treatment of HIV infection over 48 weeks: a randomized noninferiority trial. Lancet 2006; 368: 476-82.

[15] Kozal MJ, Kroodsma K, Winters MA, et al. Didanosine resistance in HIV-1 infected patients switched from Zidovudine to didanosine monotherapy. Ann Intern Med 1994; 121: 263-8.

[16] Shafer RW, Iverson AK, Winters MA, et al. Drug resistance and heterogeneous long-term virologic suppression of human immunodeficiency virus type 1 infected subjects to Zidovudine and didanosine combination therapy. The AIDS clinical trial Group $143 \mathrm{Vi}$ rology Team. J Infect Dis 1995; 172: 70-8.

[17] Picard V, Angelini E, Maillard A, et al. Comparison of genotypic and phenotypic resistance patterns of human immunodeficiency virus type 1 isolates from patients treated with stavudine and didanosine or zidovudine and lamivudine J Infect Dis 2001; 184(6): 781-4.

[18] Eron JJ, Benoit SL, Jemsek J, et al. Treatment with lamivudine, Zidovudine or both in HIV-positive patients with 200 to 500 CD4+ cells per cubic millimeter. North American HIV working Party. N Engl J Med 1995; 333: 1662-9.

[19] Campbell TB, Shulman NS, Johnson SC, et al. Antiviral activity of lamivudine in salvage therapy for multidrug-resistant HIV-1 infection. Clin Infect Dis 2005; 41(2): 236-42. 